\title{
An Alternative Promoter in Intron1 of the Renin Gene is Regulated by Glucose Starvation via Serum Response Factor
}

\author{
Philipp Lutze Heike Wanka Inga Bäumgen Doreen Staar Bianka Grunow \\ Jörg Peters
}

Institute of Physiology, University Medicine of Greifswald, Karlsburg, Germany

\section{Key Words}

Cytosolic renin $•$ Renin promoter $•$ Serum response factor $•$ Glucose starvation

\begin{abstract}
Background/Aims: Renin is known as a secretory glycoprotein that ultimately leads to angiotensin II generation. In this way renin exerts pro-inflammatory effects and promotes cardiac injury. Additional transcripts have been identified encoding for a cytosolic renin isoform that - in contrast to secretory renin - exhibits cardioprotective effects under ischemic conditions. The promoter of these transcripts is unknown. Methods: Using qRT-PCR and dualluciferase reporter assay we examined the expression and promotor activity of cytosolic renin as well as the regulation by glucose starvation in $\mathrm{H} 9 \mathrm{c} 2$ cardiomyoblasts. Results: We identified a promoter in intron 1 of the rat renin gene with two glucose starvation-sensitive regions. One region contains a binding motif for serum response factor (SRF). Under glucose depletion expression of SRF increased prior to cytosolic renin. SRF knock down selectively decreased cytosolic renin expression and attenuated the increase of cytosolic renin expression under glucose depletion. Conclusions: Transcripts encoding for secretory and cytosolic renin are differentially expressed. The low basal expression of cytosolic renin as well as its induction under ischemia-related conditions represents an efficient system regulated in accordance with its previously identified unfavorable effects under control situations but protective effects seen after myocardial infarction or glucose depletion.

\section{Introduction}

Renin is a secretory glycoprotein that, after secretion into the extracellular space, generates angiotensin (ANG) I from its only known substrate, angiotensinogen [1]. ANG I is further cleaved to ANG II, the effector peptide of the renin-angiotensin system (RAS). In the heart, ANG II increases oxidative stress, exerts pro-inflammatory effects and promotes hypertrophy [2, 3]. Thus, ANG II leads to apoptosis, necrosis, fibrosis, myocardial remodeling and hence cardiac failure $[4,5]$.

P. Lutze and H. Wanka contributed equally to the manuscript.

Prof. Dr. med. Jörg Peters

Institute of Physiology, University medicine of Greifswald

Greifswalder Str. 11 C, 17495 Karlsburg (Germany)

E-Mail joerg.peters@uni-greifswald.de 
In addition to the classical renin transcript, alternative renin transcripts have been identified in several species. In rat these transcripts were denoted as exon(1A-9)renin [E(1A-9)] [6] and in human as renin-b and renin-c $[7,8]$. These alternative transcripts lack exon1. Exon1 encodes for the signal sequence required for a co-translational transport to the endoplasmatic reticulum (ER) and thus for the sorting of the renin protein to the secretory pathway. Instead, alternative renin transcripts are translated into a truncated prorenin with use of the first in-frame UTG in exon 2. The protein lacks the pre-fragment and the first 15 amino acids of the conventional prorenin [6-8]. Consequently, the translated renin protein cannot be secreted, remains in the cytosol and is taken up by mitochondria $[6,9]$. We confirmed the translation into a cytosolic and mitochondrial located renin with enzymatic activity in vivo in transgenic rats [10]. In the rat, exon 2 is preceded by exon $1 \mathrm{~A}, \mathrm{a}$ short sequence derived from intron1. Up to date the function of exon1A is unknown. It does not contain an in-frame AUG and therefore, cannot code for an alternative 5' extension of the renin protein.

In the rat heart, the expression of cytosolic renin was increased after myocardial infarction [11]. In addition, glucose deprivation caused an increase of the transcript levels of cytosolic renin in cardiac H9c2 cells [12]. Functionally, hearts of transgenic rats overexpressing cytosolic renin were more stable against ischemia in the ex vivo Langendorff preparation and H9c2 cells transfected with cytosolic renin were protected against necrotic cell death under glucose depletion [12]. Nevertheless, basal rates of apoptosis were increased in rat $\mathrm{H} 9 \mathrm{c} 2$ cardiomyoblasts overexpressing cytosolic renin [9]. Of considerable advantage in this respect, however, endogenous expression of cytosolic renin in these cells as well as in the rat heart is very low under basal conditions.

A promoter in intron 1 of the renin gene has been suggested that drives the expression of cytosolic renin, but such a promoter has not been demonstrated so far. Aim of the present study was therefore to characterize the unknown promoter in intron 1 of the rat renin gene and to demonstrate that this promoter is subject to regulation by ischemia related conditions such as glucose deprivation. To unravel the mechanism of regulation on renin mRNA expression, we also studied the influence of the serum response factor (SRF), which has a predicted binding site at the alternative promoter region.

\section{Material and Methods}

\section{Cell culture}

Neonatal cardiomyocyte rat H9c2 cells (ATCC, Manassas, VA, USA) were maintained in DMEM medium (Lonza, Basel, Swiss) supplemented with 10\% fetal bovine serum (PAN Biotech, Darmstadt, Germany), $100 \mathrm{U} / \mathrm{mL}$ penicillin and $100 \mu \mathrm{g} / \mathrm{mL}$ streptomycin (GIBCO, Life Technologies, Darmstadt, Germany) in a humidified incubator at $5 \% \mathrm{CO}_{2}$ and $37^{\circ} \mathrm{C}$. For PCR and functional analyses, cells were seeded in culture plates (Greiner bio-one, Frickenhausen, Germany) for 3 days. They were then exposed to siRNA-mediated SRF knock down followed by glucose depletion for another 24 hours as described previously [12].

\section{Quantitative Real-Time PCR}

RNA was extracted using the RNeasy Mini Kit (Zymo Research, Freiburg, Germany) according to the manufacturer's instructions. Quality was checked by spectrophotometry (DS-11+, DeNovix Inc, Wilmington, USA). High Capacity cDNA Kit (Life Technologies, Darmstadt, Germany) was used to reverse transcribed RNA to $\mathrm{CDNA}$, which was stored at $-70^{\circ} \mathrm{C}$. For $\mathrm{qPCR}, \mathrm{cDNA}$ was diluted in nuclease-free water. Then duplicates of 20 ng cDNA were mixed with SYBR® FAST Universal 2X Master Mix containing SYBR green dye and optimized primer pairs: for exon(1A-9)renin forward TGAATTTCCCCAGTCAGTGAT and revers GAATTCACCCCAT TCAGCAC and for exon(1-9)renin forward ATGAATTCACCCCATTCAGCand revers CCAGATGGGCGGGAGGAGGATG. For quantification of SRF transcripts we used the QuantiTect Primer Assay (Qiagen, Hilder, Germany). As housekeeping gene Tyrosine 3-Monooxygenase/Tryptophan 5-Monooxygenase Activation Protein, Zeta (YWHAZ) with the primer pairs FOR: CATCTGCAACGACGTACTGTCTCT, REV: GACTGGTCCACAATTCCTTTCTTG 


\section{Cellular Physiology Cell Physiol Biochem 2017;42:1447-1457

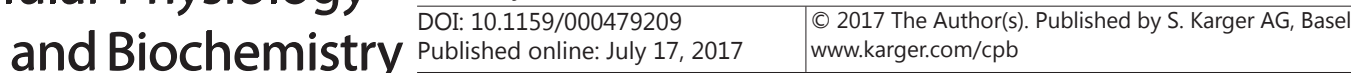

Lutze et al.: Expression of Cytosolic Renin by an Alternative Promoter

Table 1. Primer sequences for construction of deletion fragments

\begin{tabular}{lcl}
\hline Position & Direction & Primer Sequences $\left(5^{\prime}-3^{\prime}\right)$ \\
\hline$+53 \mathrm{bp}$ & Antisense & GTTCAAGCTTTGTCCTCCAATGCATC \\
$-12 \mathrm{bp}$ & Sense & TGCAGCCAGTAGCAGCTCAG \\
$-40 \mathrm{bp}$ & Sense & GTATGAGGCTCTTGCCAGGCAG \\
$-88 \mathrm{bp}$ & Sense & GCTGCAATGTCTTGGCTGGAAAA \\
$-95 \mathrm{bp}$ & Sense & CTATGAAGCTGCAATGTCTTGGCTG \\
$-228 \mathrm{bp}$ & Sense & GCTGGCGGCATCTTCCTCCAGAC \\
$-451 \mathrm{bp}$ & Sense & GGAAATAAAAAACACCCATCTGATGGA \\
$-659 \mathrm{bp}$ & Sense & CTATAGACCTGGCTGTCCTCAAACTCG \\
$-769 \mathrm{bp}$ & Sense & GCGCCCTTGCTGAGAGCATCTAG \\
\hline
\end{tabular}

was applied. The threshold cycle number (CT) in combination with the $2^{-\Delta \mathrm{CT}}$ method was normalized against the housekeeping gene YWHAZ.

\section{Promoter analysis}

For analysing the alternative renin promoter (exon1A) we used the Dual-Luciferase Reporter Assay (Promega, Mannheim, Germany) comprising expression vectors that contain the luciferase reporter gene. Different regions of interest upstream of the exon1A were amplified by PCR using rat cDNA und the primer combination listed in Table 1. After subcloning into the pGEM-TEasy vector, amplified deletion fragments were checked by sequence analyses using Sp6 and T7 primers (GATC, Germany). Correct deletion fragments were then cloned into the pGL4.10 [luc2] vector upstream of the firefly luciferase gene. Afterwards, the expression vector was introduced into the H9c2 cells using Fugen HD (Roche, Mannheim, Germany) as transfection reagent. To minimize non-relevant influences an additional vector (pGL4.74 [hRluc/TK]) containing the renilla luciferase gene was co-transfected. As negative control H9c2 cells were transfected with both the empty pGL4.10 and the pGL4.74 vectors. After an incubation time of 6 hours or 24 hours, the cells were lysed and incubated with luciferin according to the manufacturer's instructions. The enzymatic activity of luciferases was measured consecutively on a luminometer (LB96V, EG \& G Berthold, Germany). The luciferase activity of the promoter-firefly luciferase constructs was then normalized to the renilla luciferase activity derived from the pGL4.74-TK vector and expressed as relative fold increase in comparison to the promoter-less pGL4.10 basis vector.

\section{SRF downregulation by SiRNA}

Downregulation of SRF in H9c2 cells was accomplished with the RNA interference method using 20 and $40 \mathrm{nmol} / \mathrm{L}$ Stealth $\mathrm{RNAi}^{\mathrm{TM}}$ siRNA Duplex oligoribonucleotides to SRF according to the manufacturer's instructions (Thermo Fisher Scientific, Dharmacon, Schwerte, Germany). Briefly, transfection was performed for $6 \mathrm{~h}$ with the indicated siRNA and scramble control siRNA using OptiMEM (GIBCO, Life Technologies, Darmstadt, Germany) as transfection medium and polyethyleneimine (1 $\mu \mathrm{g} / \mu \mathrm{L})$ (SigmaAldrich, Taufkirchen, Germany) as transfection reagent. Following a varying incubation period of 1 to 3 days, transcript abundance and cell viability were quantified.

\section{Cell-based ELISA to the serum response factor}

The calorimetric cell-based SRF ELISA kit was performed according to manufacturer's instructions (LSBio LifeSpan Biosciences, Eching, Germany). Briefly, 20,000 H9c2 cells were plated onto a 96 well plate and cultured for 2 day at $37^{\circ} \mathrm{C}$ in the $\mathrm{CO}_{2}$ incubator. After incubation of the cells in complete culture medium or glucose depleted medium for another 3, 6 and 24 hours, respectively, cell culture medium was removed, cells were washed in TBS buffer twice and fixed in $4 \%$ formaldehyde for $20 \mathrm{~min}$ at room temperature. After washing the plate 3 times with wash buffer, the cells were incubated with quenching buffer for 20 min at room temperature followed by 3 wash steps and addition of blocking buffer for 1 hour. Then, the cells were washed 3 times again and incubated overnight at $4^{\circ} \mathrm{C}$ with either the primary anti-rabbit SRF or the anti-mouse GAPDH (internal positive control) antibodies. The primary antibodies were removed by 3 times washing and cells were then exposed to the secondary HRP-conjugated anti-rabbit or anti-mouse antibodies 
for 1.5 hours at room temperature. Lastly, after 3 times washing the ready-to-use substrate was added to each well for $30 \mathrm{~min}$ at room temperature in the dark. Using the microplate reader the OD at $450 \mathrm{~nm}$ was measured immediately after adding the stop solution to each well.

Following the calorimetric measurement of HRP activity, the Crystal Violet whole-cell staining was performed to determine cell density in order to normalize SRF data to the cell count. Cristal Violet binds to cell nuclei and gives absorbance readings proportional to the cell number located in the well. Therefore, wells were washed 2 times with wash and TBS buffers followed by incubation with Cristal Violet for 30 min at room temperature. Then the solution was decanted carefully by rinsing the plate with distilled water. After air-drying for $30 \mathrm{~min}$, SDS was added to the wells for 1 hour at room temperature and absorbance was measured at $595 \mathrm{~nm}$.

\section{Statistical analyses}

Data are presented as mean \pm SEM. All statistical analyses were performed by t-test or one-way ANOVA followed by Bonferroni comparison test, respectively using Graph Pad Prism software. Differences were considered statistically significant at $\mathrm{p}<0.05\left(^{*}\right)$ and highly significant at $\mathrm{p}<0.01\left(^{* *}\right)$ and $\mathrm{p}<0.001\left({ }^{* * *}\right)$.

\section{Results}

Glucose depletion increases Ren(1A-9) mRNA- but not Ren(1-9) mRNA levels

In H9c2 cardiomyoblasts, expression of cytosolic renin (Fig. 1A) was very low but apparently higher than that of secretory renin (Fig. 1B). Under basal conditions, relative cytosolic Ren(1A-9) and secretory Ren(1-9) expressions, normalized to the housekeeper YWHAZ, accounted to $1.33 \pm 0.13 \times 10^{-4}$ and $0.57 \pm 0.04 \times 10^{-4}$, respectively. Glucose deprivation caused a highly significant 4-fold upregulation of Ren(1A-9) expression after an exposure time of 6 and 24 hours. Expression of Ren(1-9) was not influenced under these conditions.

\section{An alternative renin promoter is located in intron1 of the renin gene}

We hypothesized that a promoter region capable of driving transcription is located in intron 1 within the $5^{\prime}$ vicinity of exon1A. To test this hypothesis, we cloned a number of deletion fragments of the 5 '-flanking region of exon1A into the pGL4.10 vector and analyzed their promoter activities in H9c2 cells after 6 and 24 hours of incubation under control condition or glucose deprivation.

As shown in Fig. 2A, the 5'-flanking region from -546bp to -26bp (520bp fragment) caused a significant 2.7-fold increase in the luciferase activity after 6 hours incubation compared to the control cells transfected with the promoter-less pGL4.10 vector or to cells transfected with the shorter 109 or $157 \mathrm{bp}$ fragments. This implies that the region between -546 and $-183 b p$ could contain a minimal promoter (shaded area).

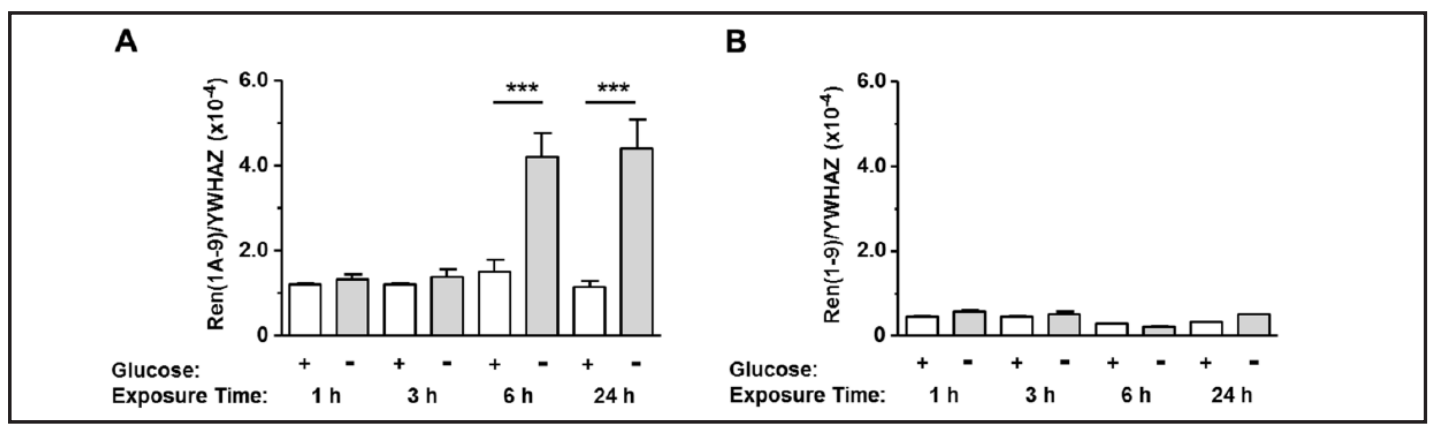

Fig. 1. Glucose starvation time dependently increased Ren(1A-9) expression in H9c2 cells. Real-time PCR was performed from $\mathrm{H} 9 \mathrm{c} 2$ cells exposed to control conditions (white columns) or glucose starvation (grey columns) for the indicated time periods. Expression pattern of (A) cytosolic Ren(1A-9) and (B) secretory Ren(1-9). Data represent mean \pm SEM of 6 experiments. Statistical analyses were performed by one-way ANOVA followed by Bonferroni comparison test with ${ }^{* * *} \mathrm{p}<0.001$.

\section{KARGER}


Fig. 2. Intron 1 of the renin gene contains an alternative promoter activated by glucose starvation. Promoter analyses were performed with deletion fragments of the 5' flanking region of exon $1 \mathrm{~A}$ cloned into two different pGL vectors upstream of the firefly luciferase gene. After transfection, H9c2 cells were exposed to (A) control conditions and (B) to glucose starvation for 6 hours. Luciferase activity was determined using the dual luciferase assay. Data represent mean \pm SEM of 6 experiments. Statistical analyses were performed by one-way ANOVA followed by Bonferroni comparison test with ${ }^{* * *} \mathrm{p}<0.001$ and ${ }^{* *} \mathrm{p}<0.01$ compared to pGL4.10 control vector and the shorter 109 and $157 \mathrm{bp}$ fragments. Shaded areas represent the presence of the minimal promoter within the $520 \mathrm{bp}$ fragment as well as the location of glucose depletion-sensitive elements within the 520 and 838bp fragments.

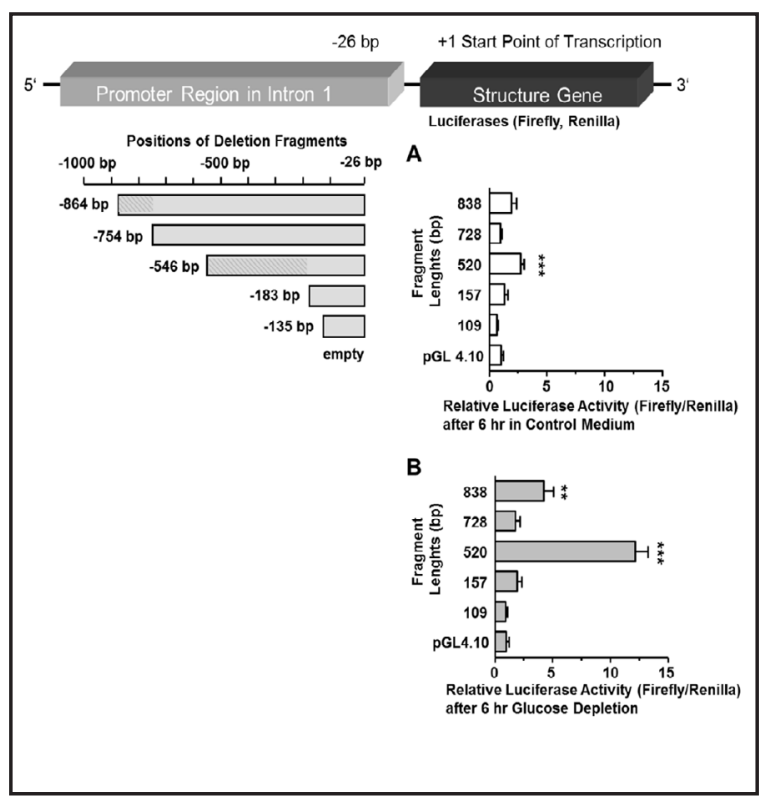

Fig. 3. A minimal promoter is located $157 \mathrm{bp}$ upstream of exon1A. The localization of a minimal promoter was determined by promotor analyses using different deletion fragments of the 5' flanking region of exon1A cloned into two different pGL vectors upstream of the firefly luciferase gene. After transfection, H9c2 cells were exposed to control conditions for 24 hours. Luciferase activity was measured using the dual luciferase assay. Data represent mean \pm SEM of 6 experiments. Statistical analyses were performed by one-way ANOVA followed by Bonferroni comparison test with ${ }^{* * *} \mathrm{p}<0.001$ and ${ }^{* *} \mathrm{p}<0.01$ compared to pGL4.10 control vector and \#\#\#p<0.001 compared to the fragments with a length of 838 and 520 bp.

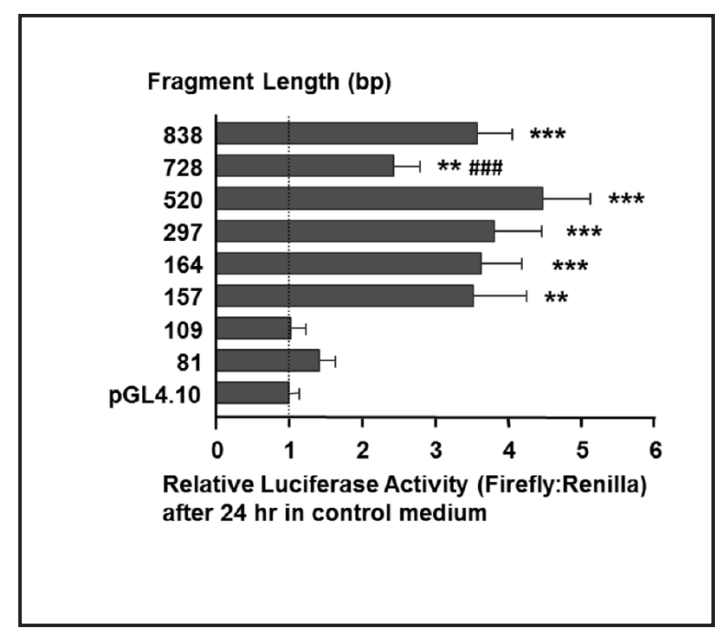

Additionally, we identified glucose deprivation-sensitive domains within the 5'-flanking promoter region of exon1A between positions -564 and $-183 \mathrm{bp}$ as well as -864 to $-754 \mathrm{bp}$ upstream of exon1A (Fig. 2B). The enhanced luciferase activity was seen after a starvation period of 6 hours, while after prolongation of glucose depletion to 24 hours this effect disappeared (data not shown).

To narrow the minimal promoter region, we extended the incubation time to 24 hours and investigated fragments closer to the transcription starting point of exon $1 \mathrm{~A}$. The results shown in Fig. 3 push the minimal promoter closer towards exon1A (157bp fragment). Interestingly, within the range of -754 to $-546 \mathrm{bp}$ a negative regulatory element(s) may exist because the promoter activity decreased highly significant. Compared to this fragment the longer $838 \mathrm{bp}$ fragment displayed a significant higher promoter activity indicating the existence of a positive regulatory element(s).

SRF upregulates the expression of Ren(1A-9)

By computational analysis using the AliBaba2.1 program, we identified numerous potential transcription factors that can bind in the alternative promoter region such as SRF. The structure of rat renin gene as presented in Fig. 4 comprises 9 exons whereby in intron1 an alternative exon1 (exon1A) is located at position 3833 to 3911 [6]. Because this exon1A 


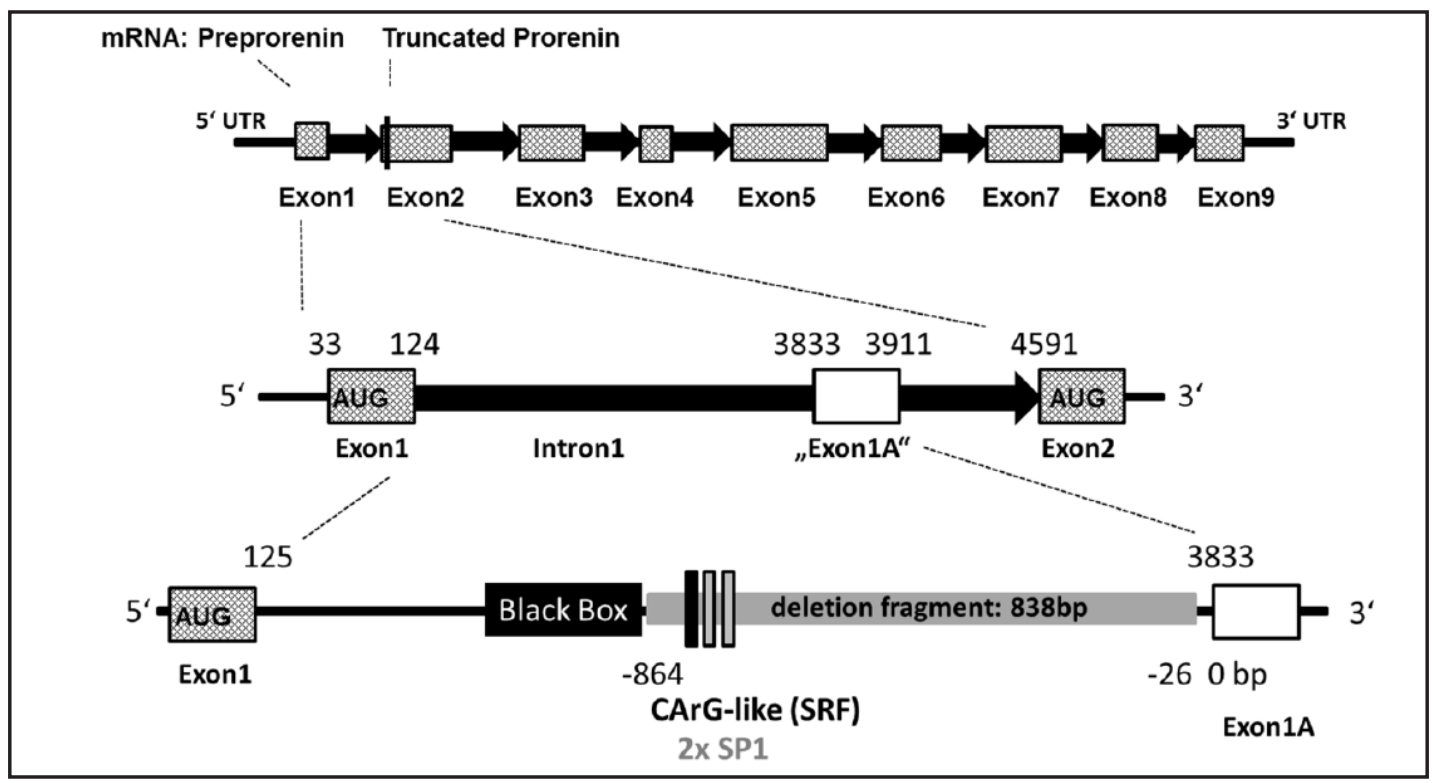

Fig. 4. Structure of the 5'-region of the renin gene. The rat renin gene comprises 9 exons (striated boxes) and 8 introns (black arrows). An alternative "exon1" (exon1A) is located within intron1. Numbers denote the positions of exon1, exon1A and exon2 in the genomic sequence [6]. Upstream of exon1A closed to the black box one SRF binding site (CArG-like sequence) and two specific protein1 (SP1) binding sites were identified by computational analysis using the AliBaba2.1 program.

\section{A}

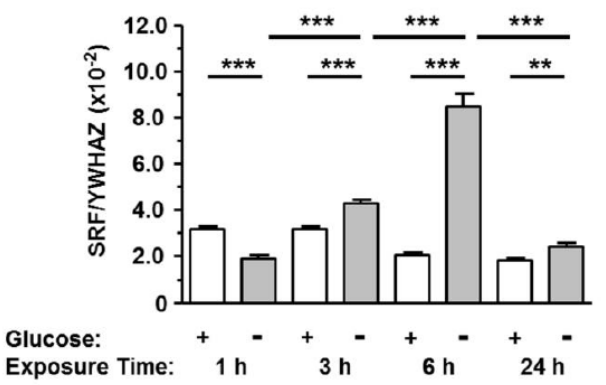

B

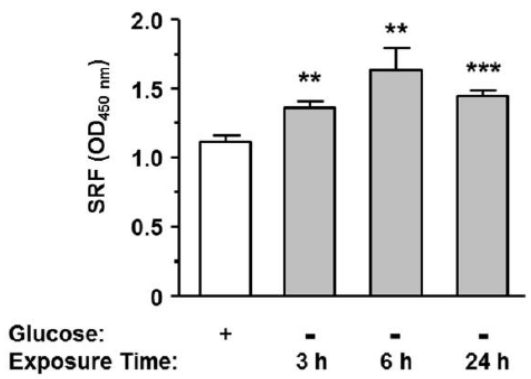

Fig. 5. Relative SRF expression increased time dependently in response to glucose starvation. Real-time PCR was performed from $\mathrm{H} 9 \mathrm{c} 2$ cells exposed to control conditions or glucose starvation for the indicated time periods. (A) Expression pattern of SRF mRNA and (B) SRF protein. Data represent mean \pm SEM of 6 and 9 experiments. Statistical analyses were performed by one-way ANOVA followed by Bonferroni comparison test with ${ }^{* * *} \mathrm{p}<0.001$ and ${ }^{* *} \mathrm{p}<0.01$.

lacks the AUG sequence within the reading frame the translation starts with exon 2 resulting in a truncated prorenin. Our described promoter analyses were performed with different fragments covering the range between $26 \mathrm{bp}$ and $864 \mathrm{bp}$ upstream of exon1A. Close to the black box we identified one serum response element (CArG-like sequence) and two specific protein 1 binding sites.

To test the hypothesis that SRF regulates the expression of the alternative renin transcript, we investigated time dependently the expression pattern of SRF itself. Here, we observed a significant upregulation of SRF already 3 hours after glucose deprivation and reaching a maximum after 6 hours, which was followed by a significant reduction 18 hours later (Fig. 5A). Compared to the expression pattern of Ren(1A-9) as seen in Fig. 1, upregulation of SRF mRNA by glucose starvation occurred before that of Ren(1A-9). Simultaneously to the mRNA expression, we also found a glucose starvation-induced increase of SRF protein 


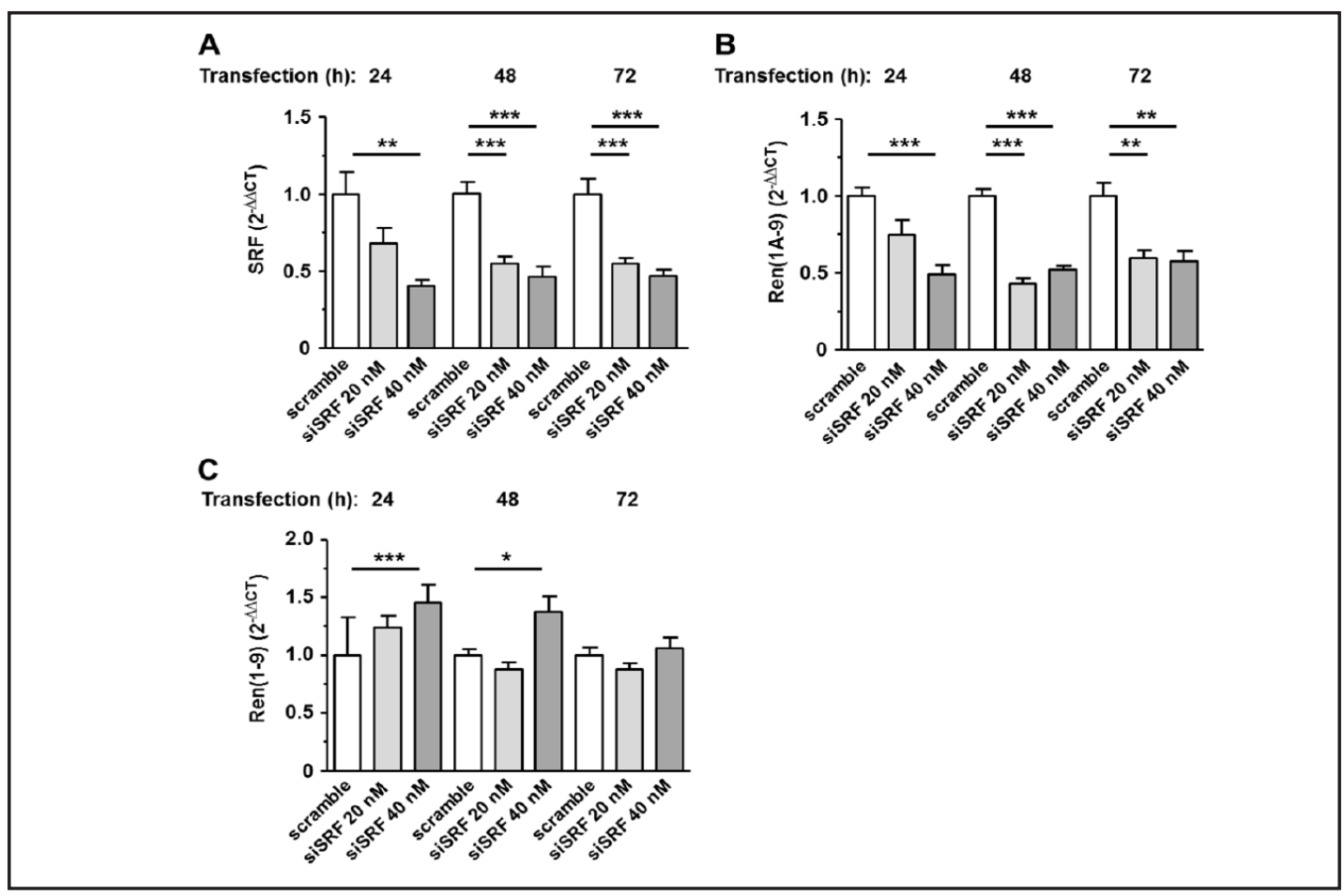

Fig. 6. Ren(1A-9) expression decreased in response to SRF knock down. Real-time PCR was performed from H9c2 cells exposed to siRNA-mediated SRF knock down for the indicated time periods. (A) Time dependent efficiency of SRF knock down normalized to the housekeeping gene YWHAZ and the scramble control. (B) Expression pattern of cytosolic Ren(1A-9) and (C) secretory Ren(1-9) each normalized to the housekeeping gene YWHAZ and the scramble control. Data represent mean \pm SEM of 6 experiments. Statistical analyses were performed by one-way ANOVA followed by Bonferroni comparison test with ${ }^{* * *} \mathrm{p}<0.001,{ }^{* *} \mathrm{p}<0.01$ and $* \mathrm{p}<0.05$.

expression (Fig. 5B). Starting 3 hours after glucose deprivation SRF protein expression remained upregulated up to 24 hours.

To demonstrate that SRF indeed regulates Ren(1A-9) expression, we next performed a SRF knock down by siRNA followed by the analyses of SRF and renin expressions to determine knock down efficiency and to confirm an interrelationship (Fig. 6). Efficiency of knock down was normalized to both the housekeeping gene YWHAZ and the scramble control. Using 20 nM siRNA the efficiency of SRF knock down accounted to $68 \pm 10 \%, 55 \pm 4 \%$ and $55 \pm 3 \%$ 24 hours, 48 hours and 72 hours post transfection, respectively. $40 \mathrm{nM}$ siRNA decreased the SRF expression to $40 \pm 4 \%, 46 \pm 7 \%$ and $47 \pm 4 \%$ in the indicated time period (Fig. 6A). In comparison, Ren(1A-9) expression decreased as well (Fig. 6B), whereas the expression of Ren(1-9) mostly remained unchanged or even increased (Fig. 6C), indicating a possible selective interrelationship between the transcription factor SRF and the expression of cytosolic Ren(1A-9).

In a next step, we analysed whether SRF mediates the glucose starvation-induced increase in $\operatorname{Ren}(1 A-9)$ expression in addition to its effect on basal expression. Therefore, we investigated the effect of glucose starvation on the expression of renin isoforms in control and SRF-depleted H9c2 cells. Fig. 7A shows that SRF knock down was successful under basal conditions but also under glucose starvation. SRF expression decreased significantly from $6.17 \pm 0.70 \times 10^{-3}$ and $8.00 \pm 0.54 \times 10^{-3}$ in the corresponding controls to $3.86 \pm$ $0.44 \times 10^{-3}$ in siRNA-treated cells. After glucose deprivation, SRF expression decreased from $10.03 \pm 0.99 \times 10^{-3}$ and $11.21 \pm 1.03 \times 10^{-3}$ in controls to $3.62 \pm 0.35 \times 10^{-3}$ in siRNA-treated cells, indicating an efficient SRF knock down under starvation conditions. Concerning renin expression, SRF knock down caused a significant reduction of Ren(1A-9) mRNA levels from 


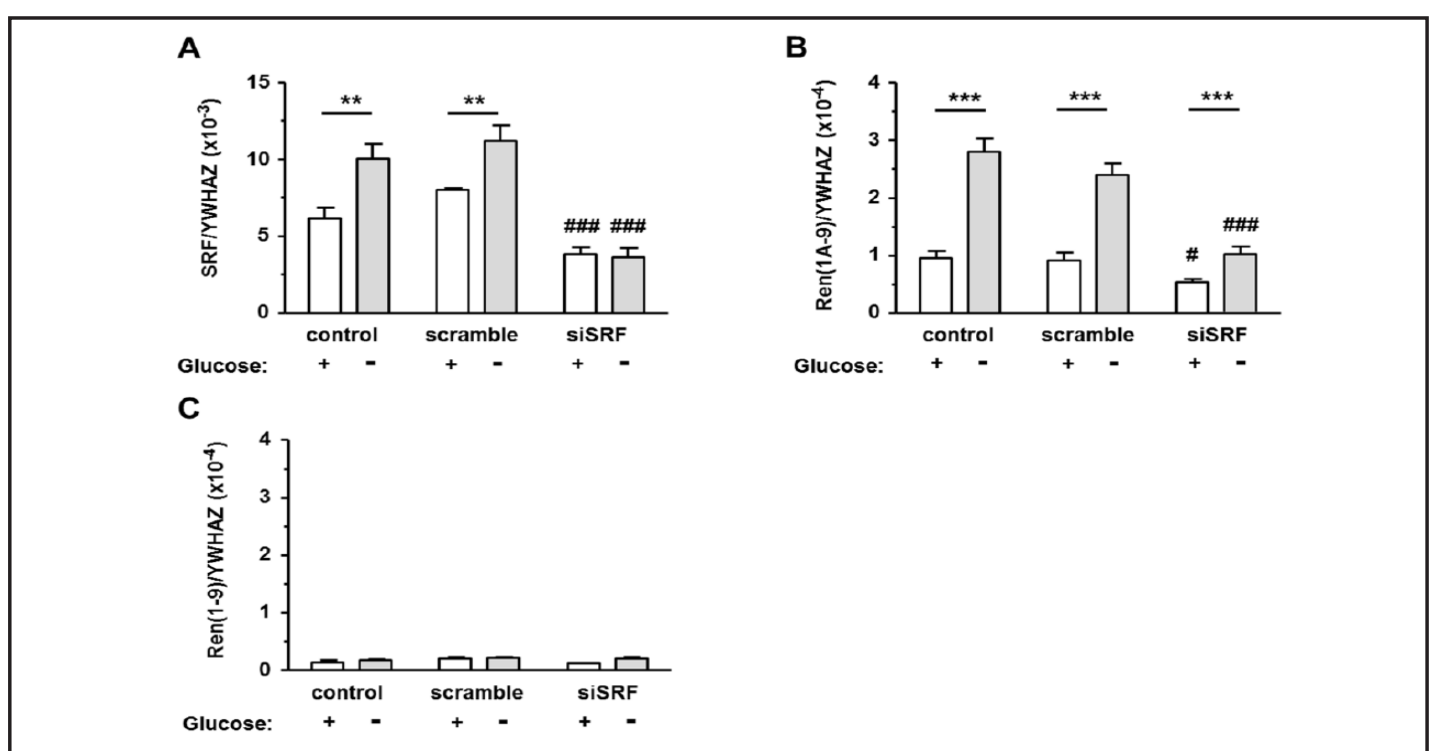

Fig. 7. SRF knock down attenuated the increase of Ren(1A-9) expression in response to glucose starvation. Real-time PCR was performed from H9c2 cells exposed to siRNA-mediated SRF knock down for 24 hours followed by culture under control conditions or glucose starvation for another 24 hours. (A) Relative SRF expression in response to glucose starvation and siRNA-mediated SRF knock down. (B) Expression pattern of cytosolic Ren(1A-9) and (C) secretory Ren(1-9) in dependence on the indicated conditions. Data represent mean \pm SEM of 7 experiments. Statistical analyses were performed by one-way ANOVA followed by Bonferroni comparison test with ${ }^{* * *} \mathrm{p}<0.001,{ }^{* *} \mathrm{p}<0.01$ and ${ }^{*} \mathrm{p}<0.05$.

$0.95 \pm 0.12 \times 10^{-4}$ and $0.94 \pm 0.12 \times 10^{-4}$ in controls to $0.54 \pm 0.06 \times 10^{-4}$ in siRNA-treated cells (Fig. 7B). The glucose starvation-mediated increase of Ren(1A-9) expression (2.81 \pm $0,22 \times 10^{-4}$ and $2.41 \pm 0.19 \times 10^{-4}$ in controls) was retarded to $1.00 \pm 0.13 \times 10^{-4}$ in siRNA-treated cells. Secretory Ren(1-9) mRNA levels were not influenced neither under basal conditions nor under glucose starvation confirming the selective interference with the alternative promoter of Exon1A (Fig. 7C).

\section{Discussion}

In the last years, important advances were made in understanding the expression and regulation of the transcripts encoding for secretory renin [13, 14]. Considerably less is known about the regulation of the transcripts encoding for cytosolic renin. We hypothesized that an alternative promoter exists in intron 1 of the renin gene that drives expression of the alternative renin transcript [E(1A-9) in rat]. In the present study, we firstly identified the alternative promoter in intron 1 of the rat renin gene. This promoter drives the expression of cytosolic Ren(1A-9) and can be activated by glucose depletion. Secondly, we identified at least two glucose dependent regulatory regions $5^{\prime}$ of the promoter. Thirdly, we provide evidence that serum response factor (SRF) is induced by glucose depletion and takes part in the regulation of cytosolic Ren(1A-9), but not of secretory Ren(1-9).

In more detail, using the luciferase reporter gene expression assay we now identified a minimal promoter which is located 135 to $183 \mathrm{bp}$ (157bp fragment) upstream of exon $1 \mathrm{~A}$. The promoter exhibited only weak responses in the luciferase reporter assay. However, the same holds true for the known promoter of secretory renin $[15,16]$. For the classical promoter of Ren(1-9) five inhibitory- and two stimulatory elements have been detected [17]. However, those elements are far 5' of the alternative promoter for Ren(1A-9). They unlikely play a role in Ren(1A-9) regulation, since the compositions of the promoters are completely different (see below). The weak promoter activity for $\operatorname{Ren}(1 A-9)$ is well reflected by the poor basal 
expression of Ren(1A-9) transcripts in H9c2 cells. Furthermore, it may explain the lacking response in the luciferase assay under basal conditions after an incubation time of 6 hours. Thus, only the prolongation of incubation time to 24 hours was sufficient to accumulate enough luciferases and to record completely the activity of the minimal promoter.

By computational analysis, we could neither find a classic TATA (5' TATAAAA 3') box nor an INR (TCA(G/T)T(T/C)) core promoter element in the region immediately flanking the exon $1 \mathrm{~A}$, indicating a TATA- and INR-less promoter. Such promoters occurring in nearly $46 \%$ of human genes are enriched in specific protein 1 (SP1) binding sites [18]. Indeed, in the $864 \mathrm{bp}$ upstream of the exon1A we identified 10 possible binding sides for SP1 with help of the transcription factor binding side program AliBaba2.1. SP1, however, appeared not to be regulated by glucose depletion in our hands (data not shown). TATA-less genes are frequently involved in basic housekeeping processes, while TATA-containing genes tend to be highly regulated by extracellular stress-related responses $[18,19]$. Despite TATA-less, the exon $1 \mathrm{~A}$ promoter was regulated by extracellular stress signals especially in response to glucose deprivation as well documented by the luciferase assay and the upregulation of the Ren(1A-9) expression. Here, we found two glucose deprivation-sensitive domains in the 5 ' upstream promoter region that could explain the observed upregulation of $\operatorname{Ren}(1 A-9)$ expression. Although the promoter activation by glucose deprivation was limited in time to 6 hours and disappeared at 24 hours, we observed a selective upregulation of $\operatorname{Ren}(1 \mathrm{~A}-9)$ at both time points. This discrepancy could be attributed to a stabilization of the renin mRNA over the indicated time period.

SRF is an important cardiac transcription factor necessary for the regulation of genes involved in cardiac structure and function [20] but also in metabolism, ion transport, and stress responses [21]. In fibroblasts the SRF gene is rapidly but only transiently induced in response to serum stimulation [22] Thus, SRF levels reach their peak at approximately 2 hours and return to basal levels 6 hours after serum stimulation. In our system, we found a delayed upregulation of SRF transcript and protein expression starting at 3 hours and reaching a maximum at 6 hours. However for the first time, this upregulation was observed after glucose starvation which could explain the temporal discrepancy. SRF activates gene expression by binding to the serum response element which contains a consensus sequence of $\mathrm{CC}(\mathrm{A} / \mathrm{T})_{6} \mathrm{GG}$ (CArG box or a CArG-like sequence). Such a CArG-like sequence was predicted by the AliBaba2.1 program in the promoter region 761 to $771 \mathrm{bp}$ upstream of exon $1 \mathrm{~A}$, suggesting a SRF-mediated regulation of Ren(1A-9) expression. Using siRNA interference method, we demonstrate that SRF is required for the selective upregulation of Ren(1A-9) expression. However, SRF is also expressed at high level in H9c2 cells cultured under basal conditions, where Ren(1A-9) expression is very low. This implicates that SRFdependent Ren(1A-9) expression is modulated in a complex manner by (patho)physiological mechanisms during cells adapt to glucose starvation. SRF has, indeed, different co-activators such as the ets domain-containing ternary complex factors [23] and the myocardin-related transcription factors (MRTF's) [24] that modulate SRF activity. Although we did not analyse such co-activators, it is known that SRF-induced target gene expression increases for example by upregulation of MRTF-A expression during hypoxia and ischemia-reperfusion in cardiac cells [25] as well as in rat cortical and hippocampal neurons [26]. Of special interest is the fact that in immediate proximity of the SRF binding site there are also two SP1 binding sites. In this respect Spencer and Misra [27] demonstrated that SP1 can potentiate SRF-mediated gene expression. SP1 activity in turn depends on its phosphorylation following numerous extracellular stimuli [28]. However, we did not further investigate the role of these two SP1 sites yet.

To further confirm a SRF-mediated upregulation of cytosolic renin, SRF knock down should prevent or limit the upregulation of Ren(1A-9) observed after glucose starvation. Despite efficient SRF knock down, exposure of cells to glucose starvation still increased Ren(1A-9) expression considerably, albeit less strongly than in the presence of regular SRF levels. This implicates that beside SRF there are further transcription factor(s) required for upregulation of Ren(1A-9) expression during starvation. Data of the luciferase assay 
confirm this hypothesis. Thus, the 520bp construct lacking the SRF binding site exhibited also a strong response to glucose starvation. In this context, we still need to identify other transcription factor(s) that bind in this region and glucose starvation-dependently regulate Ren(1A-9) expression.

In an attempt to identify the functions of renin in the brain Lavoie et al. [29] investigated double transgenic mice expressing human cytosolic or secretory renin, each together with human angiotensinogen driven by the astrocyte-specific glial fibrillary acidic protein promoter. A significant amount of human renin could be detected in the cerebrospinal fluid of mice expressing secretory renin but not in mice expressing cytosolic renin. However, mean arterial blood pressure was significantly increased in both transgenic mice strains. Furthermore, plasma renin activity (PRA) was elevated in mice overexpressing the cytosolic renin construct. Since cytosolic renin cannot be secreted, this increase of PRA remained unexplained, but may have contributed to the increased blood pressure. In contrast, in our transgenic rats overexpressing cytosolic renin, renin levels were increased in the cytosol and mitochondria but not in the circulation. Thus, there was no effect on blood pressure in our four independently generated transgenic rat lines [10]. Later, Shinohara et al. [30] demonstrated that the specific ablation of cytosolic renin in the brain resulted in hypertension. In this study, it turned out that there was an inverse regulation of the renin transcripts. The decrease of renin-b [Ren(1A-9)] expression resulted in an increase in renin-a renin [Ren(1-9)] expression in the brain. The authors, therefore, suggest that the function of renin-b [Ren(1A-9)] consists in the inhibition of renin-a [Ren(1-9)] expression. Our own observations partially support this view. Thus, glucose depletion increased Ren(1A-9) and decreased Ren(1-9) mRNA levels [12]. However, in the present study the expression of Ren(1-9) was already very low under basal conditions (near background levels) and we could not detect any further decrease under glucose depletion. In this context, downregulation of SRF decreased expression of $\operatorname{Ren}(1 A-9)$ and at the same time increased expression of Ren(1-9). Thus, it remains an attractive hypothesis that Ren(A1-9) negatively controls Ren(1-9) expression.

Taken together, cytosolic renin seems to be part of an endogenous regulatory system whose alternative promoter in intron 1 of the renin gene is suppressed under basal conditions and partly activated by SRF under glucose starvation. This type of regulation is in accordance both with the unfavorable effect under healthy conditions as well as with the protective effects of cytosolic renin seen after myocardial infarction.

\section{Acknowledgements}

This project was supported by a grant from the German Research Foundation (J. Peters: PE 366/11-1).

\section{Disclosure Statement}

The authors declare that there are no conflicts of interest.

\section{References}

1 Hackenthal E, Paul M, Ganten D, Taugner R: Morphology, physiology, and molecular biology of renin secretion. Physiol Rev 1990;70:1067-1116.

-2 Paul M, Poyan Mehr A, Kreutz R: Physiology of local renin-angiotensin systems. Physiol Rev 2006;86:747803.

-3 Unger T: The role of the renin-angiotensin system in the development of cardiovascular disease. Am J Cardiol 2002;89:3A-9A; discussion 10A.

-4 Aoki H, Richmond M, Izumo S, Sadoshima J: Specific role of the extracellular signal-regulated kinase pathway in angiotensin II-induced cardiac hypertrophy in vitro. Biochem J 2000;347:275-284.

5 Mehta PK, Griendling KK: Angiotensin II cell signaling: physiological and pathological effects in the cardiovascular system. Am J Physiol Cell Physiol 2007;292:C82-97. 


\section{Cellular Physiology Cell Physiol Biochem 2017;42:1447-1457 \begin{tabular}{ll|l} 
and Biochemistry Published online: July 17, 2017 & $\begin{array}{l}\text { C } 2017 \text { The Author(s). Published by S. Karger AG, Basel } \\
\text { www.karger.com/cpb }\end{array}$ \\
\hline
\end{tabular}}

6 Clausmeyer S, Sturzebecher R, Peters J: An alternative transcript of the rat renin gene can result in a truncated prorenin that is transported into adrenal mitochondria. Circ Res 1999;84:337-344.

-7 Lee-Kirsch MA, Gaudet F, Cardoso MC, Lindpaintner K: Distinct renin isoforms generated by tissue-specific transcription initiation and alternative splicing. Circ Res 1999;84:240-246.

-8 Sinn PL, Sigmund CD: Identification of three human renin mRNA isoforms from alternative tissue-specific transcriptional initiation. Physiol Genomics 2000;3:25-31.

-9 Wanka H, Kessler N, Ellmer J, Endlich N, Peters BS, Clausmeyer S, Peters J: Cytosolic renin is targeted to mitochondria and induces apoptosis in H9c2 rat cardiomyoblasts. J Cell Mol Med 2009;13:2926-2937.

10 Peters J, Wanka H, Peters B, Hoffmann S: A renin transcript lacking exon 1 encodes for a non-secretory intracellular renin that increases aldosterone production in transgenic rats. J Cell Mol Med 2008;12:12291237.

11 Clausmeyer S, Reinecke A, Farrenkopf R, Unger T, Peters J: Tissue-specific expression of a rat renin transcript lacking the coding sequence for the prefragment and its stimulation by myocardial infarction. Endocrinology 2000;141:2963-2970.

12 Wanka H, Staar D, Lutze P, Peters B, Hildebrandt J, Beck T, Baumgen I, Albers A, Krieg T, Zimmermann K, Sczodrok J, Schafer S, Hoffmann S, Peters J: Anti-necrotic and cardioprotective effects of a cytosolic renin isoform under ischemia-related conditions. J Mol Med (Berl) 2016;94:61-69.

13 Morris BJ, Adams DJ, van der Weyden L: Renin gene expression: the switch and the fingers. Clin Exp Pharmacol Physiol 2001;28:1044-1047.

14 Zhou X, Davis DR, Sigmund CD: The human renin kidney enhancer is required to maintain base-line renin expression but is dispensable for tissue-specific, cell-specific, and regulated expression. J Biol Chem 2006;281:35296-35304.

15 Germain S, Bonnet F, Philippe J, Fuchs S, Corvol P, Pinet F: A novel distal enhancer confers chorionic expression on the human renin gene. J Biol Chem 1998;273:25292-25300.

16 Pan L, Black TA, Shi Q, Jones CA, Petrovic N, Loudon J, Kane C, Sigmund CD, Gross KW: Critical roles of a cyclic AMP responsive element and an E-box in regulation of mouse renin gene expression. J Biol Chem 2001;276:45530-45538.

17 Voigtlander T, Ganten D, Bader M: Transcriptional regulation of the rat renin gene by regulatory elements in intron I. Hypertension 1999;33:303-311.

18 Yang C, Bolotin E, Jiang T, Sladek FM, Martinez E: Prevalence of the initiator over the TATA box in human and yeast genes and identification of DNA motifs enriched in human TATA-less core promoters. Gene 2007;389:52-65.

19 Larsen F, Gundersen G, Lopez R, Prydz H: CpG islands as gene markers in the human genome. Genomics 1992;13:1095-1107.

20 Chai J, Tarnawski AS: Serum response factor: discovery, biochemistry, biological roles and implications for tissue injury healing. J Physiol Pharmacol 2002;53:147-157.

-21 Zhang X, Azhar G, Helms S, Burton B, Huang C, Zhong Y, Gu X, Fang H, Tong W, Wei JY: Identification of New SRF Binding Sites in Genes Modulated by SRF Over-Expression in Mouse Hearts. Gene Regul Syst Bio 2011;5:41-59.

22 Spencer JA, Misra RP: Expression of the serum response factor gene is regulated by serum response factor binding sites. J Biol Chem 1996;271:16535-16543.

-23 Johansen FE, Prywes R: Two pathways for serum regulation of the c-fos serum response element require specific sequence elements and a minimal domain of serum response factor. Mol Cell Biol 1994;14:59205928.

24 Mokalled MH, Carroll KJ, Cenik BK, Chen B, Liu N, Olson EN, Bassel-Duby R: Myocardin-related transcription factors are required for cardiac development and function. Dev Biol 2015;406:109-116.

25 Zhong Z, Hu JQ, Wu XD, Sun Y, Jiang J: Anti-apoptotic effects of myocardin-related transcription factor-A on rat cardiomyocytes following hypoxia-induced injury. Can J Physiol Pharmacol 2016;94:379-387.

26 Cao XL, Hu XM, Hu JQ, Zheng WX: Myocardin-related transcription factor-A promoting neuronal survival against apoptosis induced by hypoxia/ischemia. Brain Res 2011;1385:263-274.

-27 Spencer JA, Misra RP: Expression of the SRF gene occurs through a Ras/Sp/SRF-mediated-mechanism in response to serum growth signals. Oncogene 1999;18:7319-7327.

28 Pages G, Pouyssegur J: Transcriptional regulation of the Vascular Endothelial Growth Factor gene--a concert of activating factors. Cardiovasc Res 2005;65:564-573.

29 Lavoie JL, Liu X, Bianco RA, Beltz TG, Johnson AK, Sigmund CD: Evidence supporting a functional role for intracellular renin in the brain. Hypertension 2006;47:461-466.

-30 Shinohara K, Liu X, Morgan DA, Davis DR, Sequeira-Lopez ML, Cassell MD, Grobe JL, Rahmouni K, Sigmund CD: Selective Deletion of the Brain-Specific Isoform of Renin Causes Neurogenic Hypertension. Hypertension 2016;68:1385-1392. 